\title{
Do EXPERIENCED PHYSIOTHERAPISTS APPLY Equal Magnitude of Force During a Grade I Central PA on the Cervical Spine?
}

\begin{abstract}
Background and purpose. Physiotherapists frequently use central posterior-anterior (PA) joint mobilization techniques for assessing and managing spinal disorders. Manual examination findings provide the basis for the selection of treatment techniques. From the literature it is evident that the level of reliability varies when physiotherapists perform different mobilization techniques. Repeatability of mobilization techniques is important for better physiotherapy management.
\end{abstract}

The aim of the study was to determine whether experienced physiotherapists apply equal magnitude of force during a grade I central

\section{Conradie M, MScPhysio(Stell) ${ }^{1}$ Smit E, HonsBScPhysio (Stell) ${ }^{1}$ Louw M, BScPhysio (Stell) Prinsloo M, BSc Physio (Stell) Loubser L, BSc Physio (Stell) Wilsdorf A, BSc Physio (Stell)}

${ }^{1}$ Lecturer, Department of Physiotherapy, Faculty of Health Sciences, Stellenbosch University. PA mobilisation technique on the cervical spine. Another aim was to determine the variation in the magnitude of force applied by each individual physiotherapist.

Subjects. Sample of convenience, consisting of sixteen $(n=16)$ selected qualified physiotherapists with experience in Orthopaedic Manual Therapy.

Methods. A grade I central PA was performed on the Flexiforce TM sensors positioned on C6 of the same asymptomatic model to measure the applied magnitude of force. Two separate measurements, each lasting 30-seconds, were obtained. Results. The average maximum peak force applied by the majority of physiotherapists (87.5\%) was between $10.95 \mathrm{~g}$ and $72 \mathrm{~g}$. The difference in the forces applied for the two measurements ranged between $0.64 \mathrm{~g}$ and $24.4 \mathrm{~g}$. The Bland Altman scatterplot determined the mean of the difference between measurement one and two, calculated for the group, was zero. When comparing the two measurements, little variation was noted in the forces applied, as well as the coefficient of variation for each physiotherapist.

Conclusion and Discussion. Current results demonstrated good intra-therapist and moderate to good inter-therapist repeatability. Further research is required to generalize results.

KEY WORDS: JOINT MOBILISATION, POSTERO-ANTERIOR, INTRA-THERAPIST, INTER-THERAPIST, RELIABILITY.

\section{INTRODUCTION}

Passive accessory movements (PAMs) are defined as those movements, which a person cannot perform actively or voluntarily but that can only be performed by an external force such as a physiotherapist (Maitland 1993). PAMs can either be gliding movements in a posterior, anterior, medial or lateral direction, or

\section{CORRESPONDENCE TO:}

Marlen Conradie

Department of Physiotherapy

Faculty of Health Sciences

Stellenbosch University

PO Box 19063, Tygerberg 7505

Tel: (021) 938-9300

Fax: (021) 931-7810

E-mail: mc6@sun.ac.za distraction or compression of the joint surfaces. Since these accessory movements occur during all physiological movements, it is important for physiotherapists to examine PAMs. Limited accessory movements frequently affect physiological movements (Petty \& Moore 1998). In patients presenting with spinal disorders, the examination of passive accessory intervertebral movements (PAIVMs) can assist physiotherapists to localise the symptomatic joint and/or to identify segments of the spine with joint motion abnormality by distinguishing between normal, hypo- or hypermobility (Jull 1994). These manual examination findings provide the basis for the selection of treatment techniques.

Central postero-anterior (PA) mobilisation techniques are frequently used for the assessment and management of spinal disorders. Clinically, this technique is performed by applying a PA force to the spinous process of the target vertebra that is suggested to result in an anterior translation movement relative to the adjacent vertebra (Maitland et al 2001). Passive mobilisation techniques have been graded ranging from grade I to IV (Maitland 1993). A grade I mobilisation technique is defined by Maitland (1993) as "a small-amplitude movement performed near the starting position of the range", outside the onset of resistance, also known as R1. Additionally, he qualifies a grade I as follows: "a fitting description is to say that if a fly were between the therapist's thumbs and the spinous process, it would not be squashed by the technique" (Maitland 
1993). Even though it is hard for some clinicians to believe that such gentleness can be effective in treatment, Maitland (1993) suggested that when pain severely limits movement, a grade I mobilization technique can be effective. It can be argued that a grade I does not necessarily move the joint surfaces, but pain can possibly be relieved by stimulating the superficial proprioceptors due to soft tissue mobilisation or even by touching the skin.

Connective tissue exhibits the common property of viscoelasticity (Norkin \& Levangie 1992). Viscosity refers to a material's ability to dampen shearing forces. Elasticity refers to the tissue's ability to return to its original state following deformation, after the deforming load has been removed. Viscoelastic tissues are capable of undergoing deformation when forces are applied and returning to their original state following the removal of the force. Under normal conditions, viscoelastic tissues do not return to their original state immediately. When viscoelastic tissue is subjected to a constant tensile or compressive load it deforms and continues to deform even if the load remains constant, known as creep. An increase in the magnitude of the applied force tends to increase the rate of creep because length changes or deformation are directly proportional to the magnitude of the applied forces (Norkin \& Levangie 1992). Viscoelastic materials do not store all of the energy that is transferred to them when they are deformed. When a force is applied and then removed, some of the transferred energy may be lost in the form of heat, also called hysteresis, and therefore the tissue may not return to its original length. Connective tissue deformation is also dependent on the rate and duration of loading. Generally the higher the rate and the longer the duration of the applied force, the greater the deformation, since creep and hysteresis are rate and time dependent (Norkin \& Levangie 1992). For the current study a grade I mobilisation technique was selected because this technique should be performed in the range of movement available before the onset of resistance (R1). This should minimise the possible effects of creep and hysteresis deformation due to repeated loading and unloading of the viscoelastic connective tissue of the same model when applying oscillatory passive mobilisation techniques.

However, Shirley et al (2002) demonstrated that connective tissue had a short-lived elongation response during repeated loading of the lumbar spine with a PA force, using a mechanical device, on pain-free models. According to these authors, the connective tissue returned to their pre-testing state in less than 5 minutes. Even though the specific grades of movement used were not mentioned, the applied forces ranged between 2 and 90 Newtons. According to Harms et al (1999) forces between 9 and 30 Newtons correspond to the range of forces used by physiotherapists during grade I and II mobilisations using Maitland's grading system (Maitland 1993; Maitland et al 2001). Higher forces between 165 and 190 Newtons were measured during the performance of grade III and IV PA mobilisation techniques on L3. It seems thus that it is possible to investigate the magnitude of force applied during grade I and II mobilisation techniques on the same model as long as adequate resting periods are allocated between test sessions.

In practice, many physiotherapists base the magnitude of force used for the different grades of mobilisation techniques on the interpretation of Maitland's (1993) definitions. The grading system is dependent on good palpatory skills to determine the onset of resistance (R1). For objective investigation it is therefore necessary to accurately quantify the magnitude of force, instead of depending on the therapists' perception of the onset of resistance (R1). Different mechanical devices have been developed for research purposes, in order to measure the magnitude of forces applied during passive mobilization techniques, both to the human spine and to a spinal simulator. Conflicting results regarding the inter- and intra-therapist reliability of passive mobilisation techniques were found in the literature (Binkley et al 1995; Inscoe et al 1995; Lindsay et al 1995; Simmonds et al 1995; Smedmark et al 2000; Smit et al 2003). In the study by Simmonds et al (1995), grades I-IV central PA mobilization techniques were performed by 10 physiotherapists, each with a minimum of 7 years clinical experience, on a spinal mobilization model representing three stiffness conditions by using one to three springs. The results indicated that although the magnitude of applied peak force varied with the grade of mobilisation and with the degree of stiffness, there was a large range of variability between therapists. Poor inter-therapist agreement on mobility judgement was also found in the study by Binkley et al (1995) when six experienced physiotherapists, who frequently used manual therapy techniques, evaluated lumbar PA accessory mobility on 6-levels for 18 patients with lower back pain. In addition, Smit et al (2003) found poor inter-therapist reliability regarding the applied magnitude of force when 20 third year and 20 fourth year physiotherapy students performed a grade I central PA mobilization on the spinous process of $\mathrm{C} 6$ on an asymptomatic model. In the study by Lindsay et al (1995), varying inter-therapist reliability was found when two experienced manual therapists performed various lumbo-sacral examination techniques on 8 volunteer athletes presenting with normal to mildly pathological conditions. The level of agreement varied for the different passive accessory movements, ranging from poor on the lumbar spine to excellent on the sacro-iliac joint: a central PA on the lumbar spine (14\%), a unilateral PA on the lumbar spine $(43 \%$ left, $50 \%$ right), in contrast to a unilateral AP on the sacro-iliac joint $(75 \%$ left, $100 \%$ right). When two experienced physiotherapists independently assessed passive physiological intervertebral movements (PPIVMs) on 6 segments of the lumbar spine during forward bending on 6 volunteers experiencing lower back pain symptoms (Inscoe et al 1995), better intra-therapist reliability (67\% and $75 \%$ respectively) was demonstrated in comparison to inter-therapist reliability (49\%).

In contrast to the above, Smedmark et al (2000) showed relatively high percentage agreements (ranging between $70 \%$ and $87 \%$ ) between two experienced therapists when assessing passive physiological intervertebral movements (PPIVMs) on 61 patients with cervical problems. Despite the high percentage 
agreement, the Kappa coefficients varied between 0.28 and 0.43 , suggesting fair to moderate inter-therapist reliability. Lindsay et al (1995) also indicated higher percentage agreement between therapists when assessing PPIVMs (ranging between $57 \%$ and $86 \%$ ) in comparison to PAIVMs (ranging between 14\% and $50 \%$ ) on the lumbar spine.

From the studies above, it is evident that the level of reliability varies when physiotherapists perform different mobilisation techniques on different regions of the spine, even though direct comparison between the different studies is difficult due to the variability in the methodologies. In summary, it seems that a higher level of agreement was achieved when PPIVMs were performed in comparison to PAIVMs. Mobilisation techniques performed on the sacro-iliac joint demonstrated better reliability, followed by techniques on the lumbar spine and lastly techniques on the cervical spine. Intra-therapist reliability was mostly better than inter-therapist reliability.

The overall aim of the current study was to determine whether experienced physiotherapists apply equal magnitude of force during a grade I central PA mobilisation technique on the cervical spine. An additional aim was to determine the variation in the magnitude of force applied by each individual physiotherapist between two repetitions of the same technique. This topic was chosen in order to attempt to quantify the magnitude of force applied during a grade I central PA.

\section{METHODOLOGY \\ Subjects}

A sample of convenience, consisting of qualified physiotherapists with a special interest in Orthopaedic Manual Therapy (OMT) was selected from a list of candidates who had successfully completed the OMT 1 course. After telephonic consultation, sixteen $(n=16)$ therapists who fulfilled the inclusion criteria, gave informed consent (14 female, 2 male).

Inclusion criteria for the subjects were:

- Currently registered at the Health Professional Council of South Africa (HPCSA) as physiotherapists
- Practicing in the Cape Peninsula

- Completed the OMT 1 course successfully

- Performed Maitland manual therapy techniques on the vertebral column on a weekly basis

Subjects were excluded if they treated less than four orthopedic patients presenting with vertebral column conditions per week.

\section{Equipment}

The measurement tool, as used by Smit et al (2003), consisted of two independent variable resistance transducers (FlexiForceTM, Tekscan, South Boston, MA) to measure the magnitude of force executed by each thumb. The measurement tool was calibrated daily prior to testing. The ultra-thin sensors remained stable over the testing period. Detailed description of the tool can be found in previous publication (Smit et al 2003).

\section{Procedure}

After conducting a pilot study using four qualified physiotherapists, two reported that the sensors displaced slightly, due to inadequate adhesion. The necessary adjustments were made to ensure firm adhesion of sensors.

The 16 physiotherapists performed a grade I central postero-anterior (PA) mobilisation technique on $\mathrm{C} 6$ of the same, 21-year old, asymptomatic female model. The model was positioned in prone. The researchers ensured a neutral cervical spine and that the sensors were firmly attached to C6. The procedure was explained to each therapist, where a standardized starting position, with each thumb placed on the corresponding sensor, was obtained. A trial period of 30 seconds was allocated in which each subject could feel or practice the grade I central PA mobilisation technique and become comfortable with the sensors. After 1 minute resting time, the first measurement was taken. The magnitude of force was captured by the FlexiForce $^{\mathrm{TM}}$ measurement tool while the subjects performed the grade I central PA mobilisation technique for 30 seconds. The oscillatory force was instantly displayed on the PC screen, which was positioned in such a way that the force data was only visible to the researcher. After another 1 minute resting period, the second measurement was obtained following the same procedure. The therapists received no visible or audible feedback while performing the procedure. Each therapist gave feedback regarding the possible influence of the sensors on the technique after the measurements were taken. Data collection was completed in three measurement sessions over a period of seven weeks.

\section{Statistical Analysis}

The average maximum peak force values applied by each physiotherapist were calculated for the first and second measurement respectively, as well as the mean maximum peak force of both measurements.

The mean values of the average maximum peak force for each physiotherapist were compared to determine whether the group of physiotherapists applied equal magnitude of force when performing a grade I central PA, thus determining inter-therapist repeatability. In addition, inter-therapist repeatability was determined by comparing the median of the average maximum peak force for the group of physiotherapists obtained for measurement one to the median for measurement two.

The calculated average maximum peak force applied by each physiotherapist for measurement one were compared to measurement two, to determine whether the magnitude of force were similar between two repetitions of a grade I mobilisation technique, thus determining intra-therapist repeatability. In addition, the coefficient of variation (CV) for each physiotherapist was calculated for both measurements independently. The individual $\mathrm{CV}$ values obtained for measurement one were compared to measurement two to determine the intra-therapist variation of the applied force.

Lastly, the Bland Altman statistic was calculated to determine the repeatability regarding the average maximum peak force when measurement one was being compared to measurement two. A scatterplot was used to indicate the difference between the measurements for each physiotherapist, the mean differ- 
ence for the group of physiotherapists, as well as the mean of the average maximum peak force for each physiotherapist. This gave an indication of both inter-therapist and intra-therapist repeatability.

Only data of the right thumb was used for this analysis, since the magni- tude of force measured by the left thumb was extremely small and thus negligible.

\section{RESULTS}

The average maximum peak force applied by each physiotherapist during both the first and second measurements while performing a grade I central PA mobilisation technique ranged between $10.95 \mathrm{~g}$ and $72.00 \mathrm{~g}$ for the majority (14 subjects, $87.5 \%$ ) of the physiotherapists (See Figure 1 and Table 1). However, the average maximum peak force applied by two of the physiotherapists $(12.5 \%)$ was notably higher than the rest of the group. Physiotherapist no.7 applied an

Table 1: The results of different variables for measurement one and two during a grade I p-a passive mobilization technique on $\mathrm{C6}$, performed by 16 physiotherapists.

\begin{tabular}{|c|c|c|c|c|c|}
\hline Physio & Measurement & $\begin{array}{l}\text { Ave max } \\
\text { peak force }\end{array}$ & $\begin{array}{c}\text { Frequency of } \\
\text { oscillations }\end{array}$ & $\begin{array}{l}\text { Standard } \\
\text { deviation }\end{array}$ & $\begin{array}{l}\text { Coefficient of } \\
\text { Variation }\end{array}$ \\
\hline 1 & 1 & 31.53 & 34 & 8.04 & 25.51 \\
\hline 1 & 2 & 32.23 & 35 & 6.48 & 20.09 \\
\hline 2 & 1 & 20.88 & 56 & 2.57 & 12.30 \\
\hline 2 & 2 & 26.74 & 60 & 3.57 & 13.34 \\
\hline 3 & 1 & 23.85 & 36 & 3.66 & 15.36 \\
\hline 3 & 2 & 21.25 & 35 & 3.16 & 14.88 \\
\hline 4 & 1 & 17.30 & 17 & 2.29 & 13.26 \\
\hline 4 & 2 & 23.76 & 19 & 3.90 & 16.42 \\
\hline 5 & 1 & 42.11 & 26 & 5.34 & 12.68 \\
\hline 5 & 2 & 33.84 & 27 & 4.31 & 12.73 \\
\hline 6 & 1 & 64.97 & 38 & 5.97 & 9.20 \\
\hline 6 & 2 & 59.98 & 38 & 7.53 & 12.56 \\
\hline 7 & 1 & 210.65 & 18 & 30.75 & 14.60 \\
\hline 7 & 2 & 209.03 & 18 & 31.06 & 14.86 \\
\hline 8 & 1 & 104.97 & 29 & 14.46 & 13.77 \\
\hline 8 & 2 & 120.51 & 31 & 9.59 & 7.95 \\
\hline 9 & 1 & 22.66 & 25 & 4.82 & 21.27 \\
\hline 9 & 2 & 24.44 & 30 & 4.55 & 18.62 \\
\hline 10 & 1 & 24.30 & 32 & 4.18 & 17.20 \\
\hline 10 & 2 & 23.67 & 35 & 2.39 & 10.09 \\
\hline 11 & 1 & 57.34 & 38 & 8.36 & 14.58 \\
\hline 11 & 2 & 59.40 & 40 & 11.64 & 19.60 \\
\hline 12 & 1 & 14.59 & 35 & 2.68 & 18.36 \\
\hline 12 & 2 & 10.95 & 36 & 0.85 & 7.72 \\
\hline 13 & 1 & 72.01 & 27 & 14.25 & 19.79 \\
\hline 13 & 2 & 50.72 & 28 & 7.27 & 14.33 \\
\hline 14 & 1 & 48.13 & 41 & 6.48 & 13.47 \\
\hline 14 & 2 & 43.57 & 39 & 8.58 & 19.70 \\
\hline 15 & 1 & 22.42 & 20 & 3.01 & 13.44 \\
\hline 15 & 2 & 46.82 & 25 & 5.26 & 11.24 \\
\hline 16 & 1 & 35.41 & 34 & 3.71 & 10.48 \\
\hline 16 & 2 & 25.27 & 31 & 2.68 & 10.62 \\
\hline
\end{tabular}


average maximum peak force of $210.65 \mathrm{~g}$ (measurement 1) and 209.03g (measurement 2), and physiotherapist no.8 applied $104.96 \mathrm{~g}$ (measurement 1) and $120.51 \mathrm{~g}$ (measurement 2). When comparing the average maximum peak force applied by each physiotherapist in measurement one to measurement two, little variation was noted. The smallest difference between the average maximum peak force applied for the two measurements was $0.64 \mathrm{~g}$ (subject 10) and the largest difference was $24.4 \mathrm{~g}$ (subject 15).

The Bland Altman scatterplot (Figure 2) indicated the repeatability regarding the average maximum peak force applied during a grade I central PA mobilization technique when comparing measurement one and two. The difference in average maximum peak force between the two measurements was calculated for each physiotherapist (y-axis), indicating that the majority of the group (14 subjects, 87.5\%) had a difference of $-20 \mathrm{~g}$ to $20 \mathrm{~g}$ (area between dotted lines, y-axis). Twelve (75\%) of these 14 subjects had a difference of $-10 \mathrm{~g}$ to $10 \mathrm{~g}$. The mean of the difference between measurement one and two, calculated for the group of physiotherapists, is represented by the solid black line (y-axis), indicating a mean difference of about zero. The mean of the average maximum peak force for both measurements was determined for each physiotherapist ( $\mathrm{x}$-axis), indicating that the majority of the group (14 subjects, $87.5 \%$ ) had a mean ranging between $10 \mathrm{~g}$ and $62 \mathrm{~g}$, with one outlier applying an average of $113 \mathrm{~g}$ and the other $210 \mathrm{~g}$.

Figure 3 compares the median of the average maximum peak force applied by the entire group during the first and the second measurement. The group's median regarding the average maximum peak force for the first measurement was $40.16 \mathrm{~g}$ and for the second measurement was demonstrated by physiotherapist no. 12 (Table 1).

Figure 4 indicates the coefficient of variation $(\mathrm{CV})$, calculated for the average maximum peak force applied by each physiotherapist for measurement 1 and 2 respectively, taking into account the standard deviation of each physiotherapist over the period of 30 seconds during the performance of grade I oscillations.

Figure 1: The average maximum peak force applied by each physiotherapist during a grade I p-a passive mobilization technique on $\mathrm{C6}$, for measurement one and two, as well as the mean for both measurements.

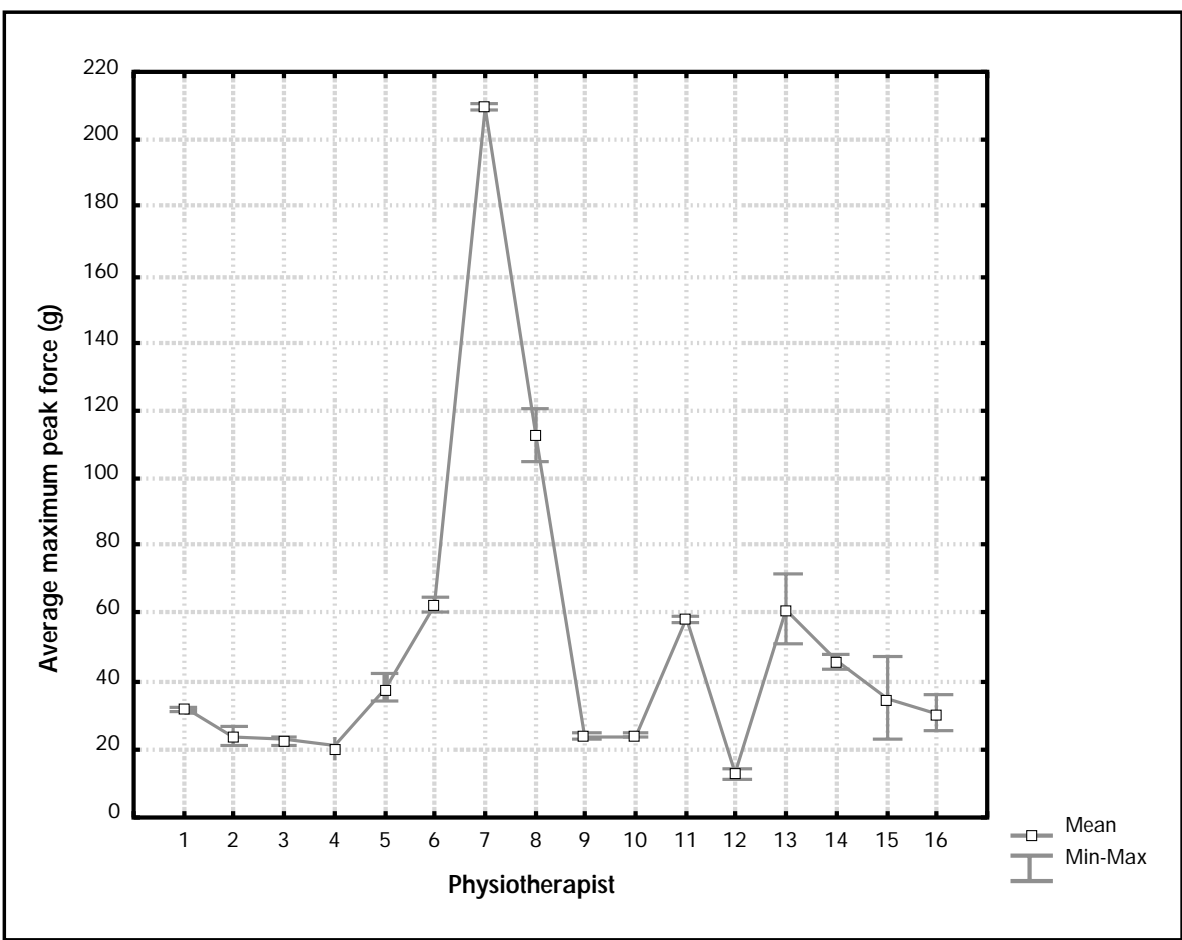

Figure 2: Bland Altman Scatterplot showing the repeatability of the average maximum peak force during a grade I $p$-a passive mobilization technique on C6, when measurement one is being compared to measurement tw 0 .

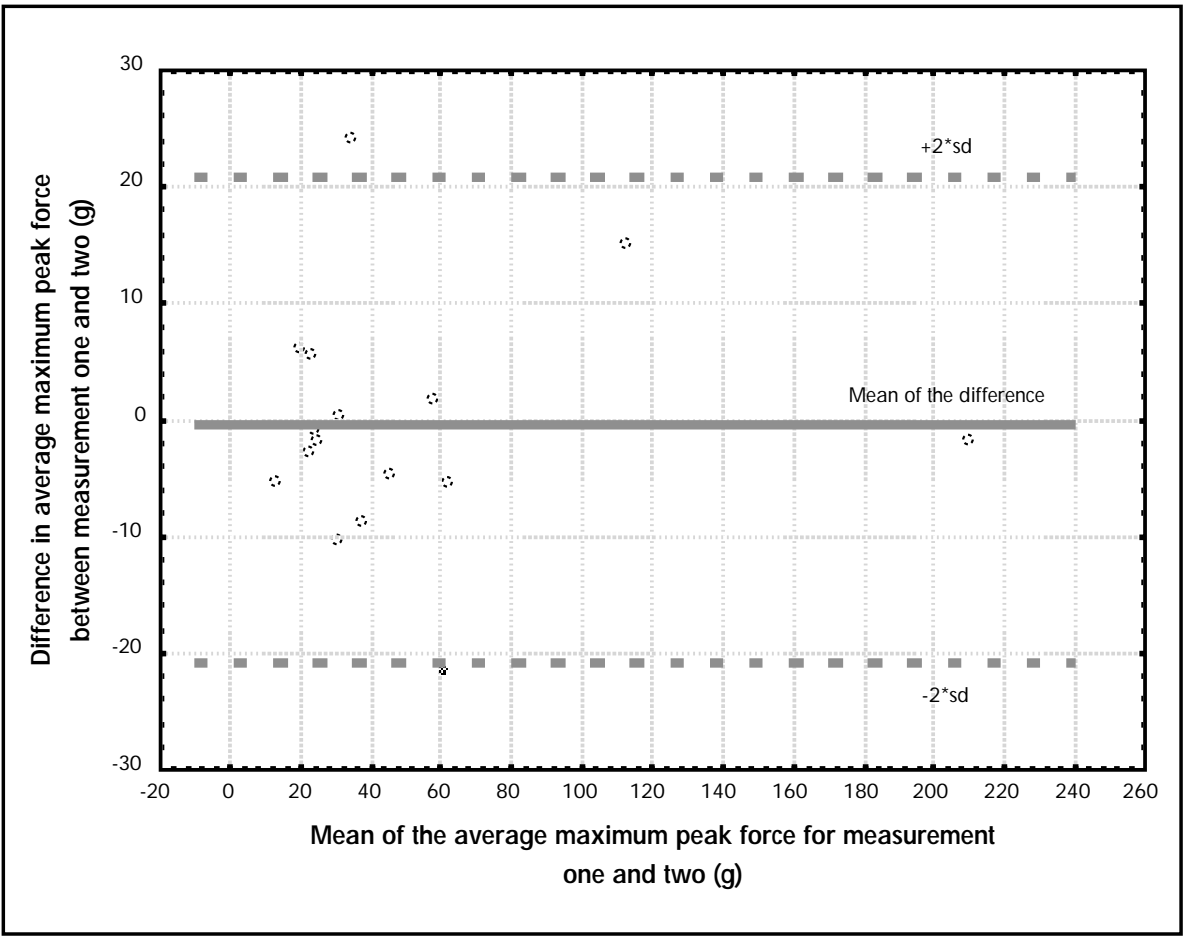

Physiotherapist no. 5, demonstrated the least difference in variation $(\mathrm{CV})$ between measurement one (12.68) and two (12.73) regarding the magnitude of force applied during the technique over
2 x 30 seconds. The greatest difference in variation between measurement one (18.36) and measurement two (7.72) was demonstrated by physiotherapist no.12 (Figure 3, Table 1). 
Figure 3: The average maximum peak force during a grade I p-a passive mobilization technique on $\mathrm{C6}$, applied by the entire group of physiotherapists during the first and second measurement.

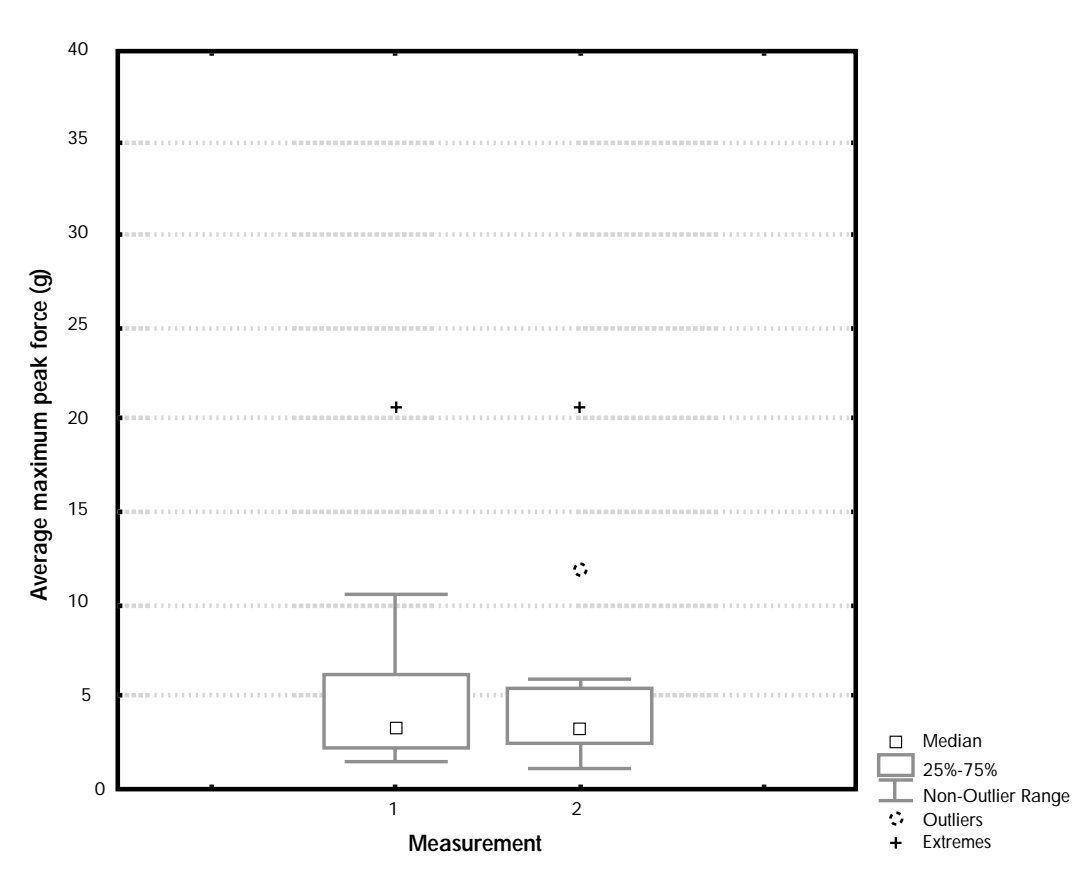

Figure 4: The coefficient of variation (CV) of the average maximum peak force during a grade I p-a passive mobilization technique on C6, applied by each physiotherapist for the first and second measurement.

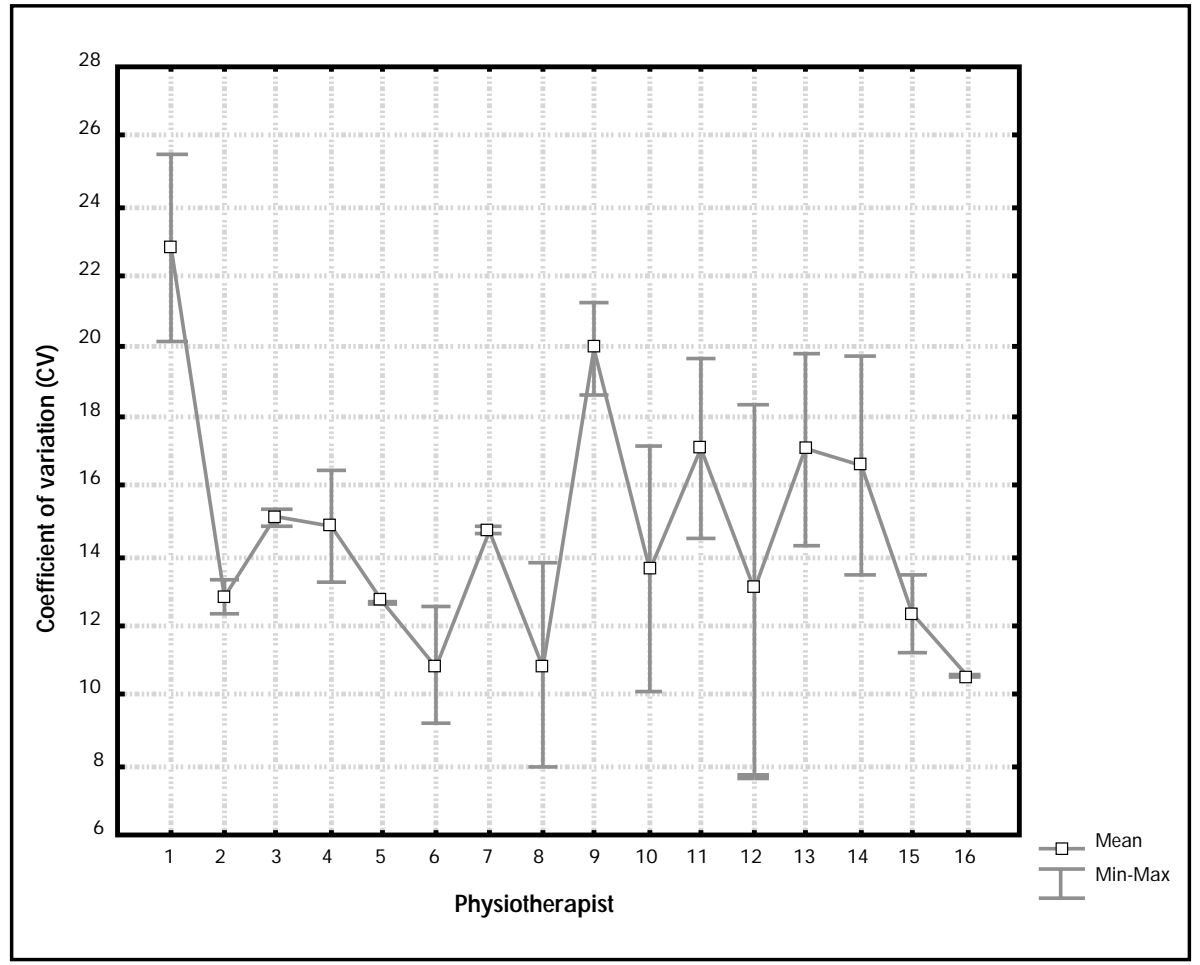

In addition to the above results, Table 1 also indicates the frequency of the oscillations performed by each physiotherapist for the grade I central PA technique during each measurement tions, performed by physiotherapist no.2, whereas the minimum frequency was 18 oscillations performed by physiotherapist no. 4 \& 7 .

The physiotherapist's feedback about the influence of the sensors on the technique indicated that only four subjects (25\%) reported a slight influence on the tactile feedback, without necessarily affecting the magnitude of force applied during a grade I central PA mobilization technique.

\section{DISCUSSION}

Before questions about effectiveness of treatment can be answered, intra- and inter-therapist reliability of passive intervertebral movements must be assessed (Gonella et al 1982). Physiological range of movement is usually measured in degrees using a goniometer, whereas accessory movements are generally measured on a scale indicating a grade of movement by using palpatory skills. However, the qualitative nature of palpatory testing lacks the quantitative precision of measurement instruments (Russell 1983). Conflicting results are found in the literature regarding the validity and reliability of this primary diagnostic tool.

The overall purpose of this study was to determine whether experienced physiotherapists apply an equal magnitude of force during a grade I central PA mobilisation technique on the cervical spine. Results indicated a relatively small variation in the forces applied by the majority of the group of experienced physiotherapists for a grade I central PA technique. Thus, moderate to good repeatability of the same technique regarding the applied magnitude of peak force amongst the group of physiotherapists (inter-therapist) was demonstrated.

An additional aim was to determine the variation in the magnitude of force applied by each individual physiotherapist between two repetitions of the same grade I central PA mobilization technique. Results indicated that little variation in the magnitude of force applied by each of the experienced physiotherapists was found when comparing measurement one with measurement two. This implies that moderate to good repeatability of the same technique was demonstrated by 
each physiotherapist. The variation in the magnitude of average maximum peak force applied by each physiotherapist for a grade I during the 30 seconds test period for both measurements was small, since the greatest difference in the coefficient of variation (CV) was 10.7 and the smallest difference in the coefficient of variation $(\mathrm{CV})$ was $0.05 \mathrm{~g}$. In general, when smaller forces are applied, a small difference in the magnitude of force between two repetitions of the same technique, will result in a larger difference in the coefficient of variation (CV) than when larger forces are performed. In this study, the physiotherapist who applied the smallest average magnitude of force, had the biggest variation in the performance of a grade I over the 30 seconds. When Maitland's definition (2001) of a grade I is considered, relatively small forces are required for grade I mobilization techniques, since a grade $\mathrm{I}$ is supposed to be performed at the beginning of range, outside the onset of resistance (R1). For this study relative small forces were expected since grade I central PA's were performed. Despite the relative small forces applied, small differences in the $\mathrm{CV}$ for the individual physiotherapists were found, further emphasizing good intra-therapist repeatability.

The clinician's sense of joint movement is a major determining factor in clinical decision-making and treatment (Russell 1983). Since physiotherapists base their goals and select interventions on the findings of palpatory testing of intervertebral movements, it is essential for the patient and profession to demonstrate acceptable repeatability in these evaluative procedures. The results of the present study demonstrated moderate inter-therapist reliability, suggesting that the same patient with the same musculoskeletal dysfunction might be "diagnosed" and treated the same by two different physiotherapists. Moderate inter-therapist reliability of assessment procedures increases our professions accountability. In addition, good to excellent intra-therapist reliability was found, implying that clinical decision making of a specific physiotherapist is based on repeatable palpatory findings.

In summary, it seems that intra-thera- pist repeatability was better than intertherapist repeatability. However, good intra-therapist repeatability is not necessarily an indication of good inter-therapist repeatability when the force applied by an individual is compared to the rest of the group. An example of this is illustrated in Figure 1 where one physiotherapist (no.7) had excellent intra-therapist repeatability, but applied much greater forces than the rest of the group.

Past studies have shown a high degree of intra- and inter-therapist variation in the performance of passive intervertebral movements. Findings of this study support the work of Inscoe et al (1995) who found better intratherapist reliability $(66.67 \%$ and $75 \%$ respectively) between two experienced physical therapists, than inter-therapist reliability (48.61\%). In addition, Gonnella et al (1982) also reported acceptable levels of intra-therapist reliability but low levels of inter-therapist reliability when five physiotherapists each evaluated the same five subjects twice. However, in both of the above studies PPIVMs were performed on the lumbar spine (Inscoe et al 1995; Gonnella et al 1982) in contrast to the current study in which grade I central PA (PAIVM) was performed on the cervical spine. Evidence therefore suggested that intra-therapist repeatability is constantly better than inter-therapist repeatability, whether passive mobilisation techniques were performed on the lumbar or cervical spine. Even though intra-therapist repeatability is very important for physiotherapy practice, it is, in isolation, not sufficient to ensure reliability of graded passive joint mobilisation techniques.

Direct comparison between similar studies is difficult due to variability in the different selected methodologies, e.g. human versus simulated spinal models, various passive intervertebral movements, including PAIVMs and/or PPIVMs, performed on different regions of the spine, assessing different aspects such as pain provocation, level of stiffness or mobility, the identification of the marked spinal level, and the use of different statistical analyses. However, when comparing different studies, the majority of studies reported poor to moderate inter-therapist reliability (Binkley et al 1995; Simmonds et al 1995; Maher \& Adams 1994; Lindsay et al 1995). In contrast, better inter-therapist reliability was found in studies performed on the cervical spine (Smedmark et al 2000; current study). From the above, it seems as if better agreement amongst therapists was found when passive intervertebral movements were performed on the cervical spine in comparison to the lumbar spine. According to Lindsay et al (1995), unilateral PAs performed on the lumbar spine had a higher percentage agreement (left $43 \%$, right $50 \%$ ) than central PAs (14\%), whereas techniques performed on the sacro-iliac joint had the best agreement (left 75\%, right 100\%). The region of the spine in which a technique is being tested could have an influence on the level of agreement.

In other similar publications, the level of continual education, years of experience in OMT, as well as the size of the sample of this current study, were comparable. The current study demonstrated (1) better agreement regarding the average maximum peak force between physiotherapists (inter-therapist repeatability) than other comparable studies, (2) that intra-therapist repeatability was better than the inter-therapist repeatability and (3) that good intra-therapist repeatability is not necessarily an indication of good inter-therapist repeatability and vice versa.

Findings of this study cannot be generalized to all the various mobilization techniques and the different grades of movement due to the small sample size, and since the reliability of only one technique was assessed, i.e. a grade I central PA, on one spinal level, C6, of the same model. More research and replication studies are therefore essential before results can be generalized. Although a limitation of this study was the assessment of a grade I technique which is seldom used in clinical practice, results have relevance regarding reliability of mobilisation techniques, enhancing the profession's accountability. Therefore, further investigation is necessary, comparing different passive intervertebral techniques performed on the different regions of the spine, on bigger samples, including determining the onset of resistance (R1), as well as different grades of 
mobilization techniques like grade III and IV, where more forces are required when moving into resistance.

\section{CONCLUSION}

Results of this investigation demonstrated good intra-therapist repeatability and acceptable inter-therapist repeatability in the magnitude of force when performing a grade I central PA on C6 by experienced physiotherapists. This study therefore provided evidence regarding the reliability of this technique when used in clinical practice, especially when performed by the same physiotherapist. Since passive intervertebral joint movements are central to assessment, treatment and re-assessment of treatment effectiveness, the reliability of these techniques is important. More research will be required before results can be generalized.

\section{REFERENCES}

Binkley J, Stratford PW, Gill C 1995 Interrater reliability of lumbar accessory motion mobility testing. Physical Therapy 75(9): 786-792
Gonnella C, Paris SV, Kutner M 1982 Reliability in evaluating passive intervertebral motion. Physical Therapy 62(4): 436-444

Harms MC, Innes SM, Bader DL 1999 Forces measured during spinal manipulative procedures in two age groups. Rheumatology 38 : 267-274

Inscoe EL, Witt PL, Gross MT, Mithell RU 1995 Reliability in evaluating passive intervertebral motion of the lumbar spine. The Journal of Manual and Manipulative Therapy 3(4): 135-143

Jull GA 1994 Examination of the articular system. In: Boyling JD, Palastanga N (ed) Grieve's Modern Manual Therapy, 2nd edn. ch 37, p 511. Churchill Livingstone, Edinburgh

Lindsay DM, Meeuwisse WH, Mooney ME, Summersides J 1995 Interrater reliability of manual therapy assessment techniques. Physiotherapy Canada 47(3): 173-179

Maher C, Adams R 1994 Reliability of pain and stiffness assessments in clinical manual lumbar spine examination. Physical Therapy 74(9): 801-811

Maitland GD 1993 Vertebral manipulation, 5th edn. Butterworth-Heinemann, Boston

Maitland GD, Banks K, English K, Hengeveld E (eds) 2001 Maitland's vertebral manipulation, 6th edn. Butterworth-Heinemann, Oxford
Norkin CC, Levangie PK 1992 Joint structure and function, 2nd edn. FA Davis, Philadelphia

Petty NJ, Moore AP 1998 Neuromusculoskeletal examination and assessment: A handbook for therapists, 1st edn. Churchill Livingstone, Edinburgh

Russell R 1983 Diagnostic palpation of the spine: A review of procedures and assessmeny of their reliability. Journal of Manipulative and Physiological Therapeutics 6(4): 181-183

Shirley D, Ellis E, Lee M 2002 The response of posteroanterior lumbar stiffness to repeated loading. Manual Therapy 7(1): 19-25

Simmonds MJ, Kumar S, Lechelt E 1995 Use of a spinal model to quantify the forces and motion that occur during therapists' tests of spinal motion. Physical Therapy 75(3): 212-222

Smedmark V, Wallin M, Arvidsson I 2000 Inter-examiner reliability in assessing passive intervertebral motion of the cervical spine. Manual Therapy 5(2): 97-101

Smit E, Conradie M, Wessels J, Witbooi I, Otto R 2003 Measurement of the magnitude of force applied by students when learning a mobilisation technique. South African Journal of Physiotherapy 59(4): 3-8

\section{Kinesiology OF THE Musculoskeletal SysteM FOUNDATIONS FOR PHYSICAL REHABILITATION}

\author{
Author: Donald A Neumann \\ ISBN 0-815-16349-5
}

$\mathrm{W}$ hat a splendid book! It certainly fills a great need for rehabilitation professionals. The text is presented in a very clear, comprehensive and logical manner and is accompanied by excellent illustrations. The book is a pleasure to read and is a very valuable source of information and a superb reference text. The book is clearly written by an authority in the field and an experienced teacher and as a result the information is presented in a thorough and easy-touse text.
The author states that the book is primarily designed for the purpose of teaching but I consider it as an essential addition to the reading list of the informed, academic practitioner.

This book consists of 15 chapters and they are divided into four sections. The first section is an introduction to kinesiology and consists of the basic structures and functions of joints and muscles. It also provides a chapter on biomechanical principles. The second section deals with the upper extremity. The axial skeleton is presented in the third section and the topic of the fourth section is the lower limb. Most chapters in sections two to four start with the morphology and functions of the bones and is followed by the anatomy and functions of the joints. The most extensive components of these sections are the discussions on the interactions of muscles and joints. Clinical examples are used extensively throughout and often lead to clearer understanding of underlying principles.

There are four distinguished contributors to this book and 37 well-known reviewers. Chapters are very well referenced and the illustrations and drawings are of a very high quality.

I would highly recommend the acquisition of this up to date textbook on Kinesiology.

Celie Eales 